\title{
NOVAS PERSPECTIVAS EM EDUCAÇÃO ALIMENTAR E
}

NUTRICIONAL

\section{Sabrina lonata de Oliveira Kathleen Sousa Oliveira}

Resumo: A crescente ênfase na temática de educação alimentar e nutricional, decorrida da estratégia Fome Zero e da Política Nacional de Alimentação e Nutrição, bem como da mudança no perfil de morbi-mortalidade da população, tornou fundamental a reflexão sobre sua configuração enquanto ação governamental. Na estratégia Fome Zero, a educação alimentar e nutricional é prevista como ação estruturante, de tal sorte que a mesma configurou-se institucionalmente no Ministério do Desenvolvimento Social e Combate à Fome (MDS) tendo como perspectiva a promoção da segurança alimentar e nutricional. Dessa forma, a Coordenação responsável pelo desenvolvimento de ações educativas nesse Ministério promoveu duas oportunidades de reflexão: uma interna, que visava estabelecer o marco institucional da educação alimentar e nutricional no MDS, e a outra no Conselho Nacional de Segurança Alimentar, com o objetivo de subsidiar o grupo de trabalho de alimentação adequada e saudável. Dessas discussões surgiram importantes recomendações e estratégias como base para o desenvolvimento de ações educativas em alimentação e nutrição tanto para o governo como para a sociedade civil. Dentre essas recomendações, destacamos a importância de que as ações educativas sejam desenvolvidas no sentido de promover a autonomia dos indivíduos, a interdisciplinaridade e a transdisciplinaridade, respeitando as culturas alimentares e valorizando a história alimentar e a diversidade regional, ao mesmo tempo em que reconheçam os saberes populares e fomentem a biodiversidade local.

Palavras-chave: Políticas públicas. Nutrição. Educação alimentar e nutricional. 
A definição da estratégia Fome Zero como prioridade do Governo Federal, coordenada pelo Ministério do Desenvolvimento Social e Combate à Fome (MDS), fundamenta-se em um conjunto articulado de políticas e recomendações que concilia ações emergenciais de acesso aos alimentos e ações estruturantes destinadas à consolidação de uma situação de segurança alimentar e nutricional no país.

Essa estratégia possui como eixos articuladores o acesso aos alimentos, o fortalecimento da agricultura familiar, a geração de renda e a articulação, mobilização e controle social, sendo que no inicio do governo federal eleito em 2001 muitas ações relativas a esses eixos ficaram sob a responsabilidade do extinto Ministério Extraordinário de Segurança Alimentar (MESA). Entre as ações do eixo "acesso aos alimentos", se destacavam diversos projetos relacionados à educação alimentar e nutricional.

Com a extinção do MESA e a criação do Ministério do Desenvolvimento Social e Combate à Fome (MDS), esses projetos foram agrupados sob uma única Coordenação, a Coordenação Geral de Educação Alimentar e Nutricional (CGEAN), que está subordinada ao Departamento de Apoio a Projetos Especiais da Secretaria Nacional de Segurança Alimentar e Nutricional.

As ações estruturantes de Educação Alimentar e Nutricional desempenham uma função estratégica para a promoção da segurança alimentar e nutricional em todas as suas dimensões, passando desde a produção até o consumo dos alimentos, considerando aspectos éticos, culturais, socioeconômicos e regionais, entre outros, na promoção de hábitos alimentares adequados e saudáveis.

Educação alimentar e nutricional sob a perspectiva da segurança alimentar e nutricional

Sabe-se que a fome e a desnutrição são flagelos existentes nas populações em estado de extrema pobreza, decorrentes da ausência ou inoperância de políticas públicas sociais que assegurem o Direito Humano à Alimentação Adequada - DHAA e a Segurança Alimentar e Nutricional - SAN como fundamentos para a garantia do direito à cidadania.

Observa-se nas últimas décadas a diminuição do déficit estatural da população decorrente da melhoria nas condições gerais de vida, como expansão do saneamento básico e no atendimento primário à saúde (Ministério da Saúde, 1990). Segundo estudo realizado pelo MDS (Brasil, 2006), especificamente na região do semi-árido brasileiro, observa-se déficit de estatura em relação à idade em $6,6 \%$ das crianças menores de cinco anos. Embora existam limitações em se comparar esses resultados com os encontrados em outras pesquisas, observa-se que ocorreu um declínio significativo de $7 \%$ ao ano. 
Por outro lado, a prevalência de sobrepeso e obesidade em crianças aumentou de 4,1\% para 13,9\%, decorrente de uma diminuição na prática de atividade física e de mudanças no padrão dietético (Wang, Monteiro, \& Popkin, 2002). Estudo recente do Instituto Brasileiro de Geografia e Estatística (IBGE, 2006) demonstra que houve um aumento considerável na proporção de adolescentes brasileiros com excesso de peso: em 1974/75, 3,9\% dos meninos e 7,5\% das meninas entre 10 e 19 anos estavam acima do peso; já em 2002/03, os percentuais encontrados foram $18 \%$ e $15,4 \%$, respectivamente.

Ainda, em relação ao perfil nutricional epidemiológico, cabe destacar que, segundo Batista Filho e Rissin (2003), a anemia permanece com prevalência elevada atingindo entre $40 \%$ a $50 \%$ dos menores de cinco anos; a deficiência de iodo encontra-se sob controle e a hipovitaminose A apresenta magnitude mais baixa que decênios anteriores.

Esse quadro epidemiológico revela uma fase de transição com predominância das doenças crônicas não degenerativas cuja morbi-mortalidade associa-se com a alimentação, à nutrição e o estilo de vida dos brasileiros. Nesse sentido, apresenta-se um quadro de insegurança alimentar com novas características em que além de problemas relacionados à carência dos alimentos temos os relacionados ao excesso e às escolhas alimentares dos indivíduos, muitas vezes inadequadas.

Nesse sentido é que a promoção da segurança alimentar e nutricional ${ }^{1}$ não pode se limitar a proporcionar à população acesso a uma alimentação, mas deve vincular a essa condição a necessidade de que os alimentos sejam também de qualidade e estejam disponíveis em condição permanente.

As práticas alimentares do indivíduo são resultadas de decisões, conscientes ou não, estreitamente relacionadas à cultura alimentar de sua região, à tradição alimentar de seu convívio social e às transformações decorridas do acesso à informação científica e popular. No entanto, no campo do conhecimento da alimentação e da nutrição, mais do que proporcionar o acesso a essas informações, torna-se fundamental estimular a autonomia dos indivíduos a fim de possibilitar que os mesmos atuem intencionalmente e com independência de influências (liberdade), o que demanda um consistente processo educativo.

É nesse propósito que a educação alimentar e nutricional, na perspectiva do DHAA e da SAN, desempenha uma função estratégica para a promoção de hábitos alimentares saudáveis.

A segurança alimentar e nutricional consiste na realização do direito de todos ao acesso regular e permanente a alimentos de qualidade, em quantidade suficiente, sem comprometer o acesso a outras necessidades essenciais, tendo como base práticas alimentares promotoras de saúde, que respeitem a diversidade cultural e que sejam ambiental, cultural, econômica e socialmente sustentáveis (Brasil, 2004). 
Projetos educativos desenvolvidos no âmbito do MDS

Até o ano de 2006, o MDS apoiou e desenvolveu diversos projetos educativos destacando-se entre eles os projetos "Educação a Mesa", "Criança Saudável, Educação Dez", "Cozinha Brasil, Alimentação Inteligente" e "Eu aprendi, eu ensinei".

O projeto Educação a Mesa foi uma parceria estabelecida entre o MDS e a Fundação Roberto Marinho, com o intuito de treinar uma rede de mobilizadores aptos a educar sobre segurança alimentar e nutricional e de elaborar material educativo para desenvolvimento de ações com a população. Para tanto, foi desenvolvido um kit pedagógico composto por: 5 obras audiovisuais de 15 minutos, 5 cadernos temáticos de capacitação, 1 caderno com atividades pedagógicas; 2 programas de televisão da série temática"Brava Gente Brasileira"; 30 interprogramas de 1 minuto cada para vinculação em televisão; CD com 12 chamadas de rádio e 2 cadernos com informativos sobre a implementação do projeto e experiências locais das ações do projeto.

O Criança Saudável, Educação Dez foi um projeto desenvolvido com várias parcerias: Editora Globo, Ministério da Saúde, Ministério da Educação, Fundo Nacional de Desenvolvimento da Educação e Correios. O objetivo do mesmo foi o de divulgar conteúdos de educação alimentar e nutricional para crianças em idade escolar e educadores. Foram produzidas cartilhas educativas como instrumentos de promoção de uma alimentação saudável, sendo 5 diferentes exemplares abordando tema de alimentação e nutrição, a saber:(i) o que é educação alimentar, (ii) vitaminas e minerais, (iii) carboidratos e proteínas, (iv) alimentação saudável, e (v) o que é obesidade. No total, foram impressos 94 milhões de cartilhas de forma a atender todos os 18 milhões de alunos matriculados nos quatro primeiros anos do ensino fundamental da rede pública brasileira.

O projeto envolve, ainda, a produção de cadernos do professor com sugestões de atividades para sala de aula e o conteúdo científico das cartilhas mais aprofundado, de forma a estimular os educadores a utilizar as cartilhas com seus alunos em sala de aula, visando a atender 700 mil professores do ensino fundamental.

O público-sujeito preferencial para as cartilhas são os escolares dos primeiros anos do ensino fundamental da rede pública, pois o material utiliza linguagem simples e próxima à utilizada pelas crianças. As cartilhas, ao fazerem isso sob a forma de histórias em quadrinhos divertidas, que têm aceitação e penetração garantidas em todas as faixas etárias por utilizarem uma comunicação basicamente visual, com pouco texto, contribuem para levar as crianças a incorporarem naturalmente novos conceitos e procedimentos em relação a seus hábitos alimentares.

O projeto Cozinha Brasil, Alimentação Inteligente é uma parceria entre o MDS e o Serviço Social da Indústria - SESI para o desenvolvimen- 
to de atividades pedagógicas de capacitação de multiplicadores e de orientação alimentar e nutricional à comunidade, utilizando como espaço didático uma cozinha experimental itinerante (unidade móvel).

As capacitações são focadas na utilização integral dos alimentos dando ênfase aos alimentos regionais e que estão em época de safra, como forma de promover uma alimentação nutricionalmente adequada e de baixo custo. $O$ projeto está implementado em todos os estados e 144 mil pessoas já participaram de seus cursos.

Por fim, o projeto Eu aprendi, eu ensinei foi desenvolvido em parceria com o Núcleo SALUS Paulista e o CREN (Centro de Recuperação e Educação Nutricional), com o intuito de incentivar a ação protagônica de estudantes do ensino médio na prevenção da desnutrição materno-infantil em suas comunidades. Em 2004 foi implementado em 11 municípios da região norte de Minas Gerais e Vale do Jequitinhonha, atuando em 53 escolas públicas estaduais e tendo como ponto forte a metodologia de mobilização social nas comunidades escolares envolvendo diretores, professores e estudantes no desenvolvimento de estratégias de intervenção social de acordo com as realidades locais.

Objetivo e princípios norteadores da coordenação geral educação alimentar e nutricional

À medida que esses projetos iam sendo executados e finalizados, diversos questionamentos surgiam tanto quanto à execução em si das atividades educativas como em relação ao próprio estado da arte da educação alimentar e nutricional. De tal forma que foram realizadas duas oficinas: a primeira, em 25 de maio de 2006, foi a "Oficina Interna da Coordenação geral de Educação Alimentar e Nutricional"; a segunda, em 08 de agosto de 2006, ocorreu no âmbito do Conselho Nacional de Segurança Alimentar e Nutricional - CONSEA e foi intitulada "Oficina de Educação Alimentar e Nutricional no Grupo de Trabalho Alimentação Adequada e Saudável".

Essas oficinas tiveram por objetivo refletir sobre diversos aspectos, a exemplo de como a educação aparece compondo a educação nutricional, como se inscreve o alimento e, consequentemente, $\mathrm{o}$ ato alimentar, nas ações de educação nutricional, qual o impacto das ações de educação alimentar e nutricional já realizadas e quais caminhos percorrer para fortalecer a educação alimentar e nutricional enquanto política de Estado.

A primeira oficina levou à construção de novo objetivo que orienta as ações de educação alimentar desenvolvidas no âmbito do MDS: promover a educação alimentar e nutricional visando à alimentação adequada e saudável no sentido de prazer cotidiano, de modo a estimular a autonomia do indivíduo e a mobilização social, valorizar e respeitar as 
especificidades culturais e regionais dos diferentes grupos sociais e etnias, na perspectiva da Segurança Alimentar e Nutricional e da garantia do Direito Humano à Alimentação Adequada.

Enquanto estratégia para o enfrentamento dos problemas alimentares e nutricionais no Brasil, a educação alimentar e nutricional proposta pelo MDS pretende ser transformadora. Para tanto, baseia-se numa perspectiva problematizadora, que ultrapasse a visão puramente instrumental e instrucional da educação, e que passe a considerá-la como a realização da pessoa.

A CGEAN compreende que a educação alimentar e nutricional deve estar voltada para todas as dimensões da segurança alimentar e nutricional, de modo a preconizar uma abordagem educacional que englobe aspectos culturais, regionais, históricos, sociais, econômicos, biológicos e afetivos, entendendo, portanto, o corpo como um todo que interage com o seu meio de modo dinâmico, interdependente e interrelacional. Uma abordagem ética que enfatize as interconexões entre o biológico, o cultural e o simbólico.

Nesse mesmo sentido, compreende-se que a educação alimentar e nutricional deve contemplar todas as fases do ciclo de vida, com respeito às especificidades dos diversos grupos sociais num processo permanente. Isso porque a aprendizagem se estabelece diariamente, em todos os momentos da vida, e "a cada novo dia não repetimos apenas os anteriores, mas os reconstruímos, refazemos" (Demo, 2006).

Já a segunda oficina resultou em diversas recomendações para a prática e a implementação de ações de educação alimentar e nutricional. Essas recomendações foram precedidas de forte reflexão sobre o estado da arte da educação alimentar e nutricional, onde ficou evidente que $o$ enfoque está na informação e que o papel da educação alimentar e nutricional não está claro. Há uma lacuna entre o discurso e a prática de educação alimentar e nutricional, as ações educativas não compreendem o comportamento alimentar e a educação está estabelecida de forma autoritária e com enfoque nos aspectos puramente biológicos da alimentação. Como recomendações dessa oficina, destacamos:

Quanto aos preceitos: (i) recomendar a utilização da expressão"educação alimentar e nutricional" ao invés de educação alimentar ou educação nutricional; (ii) desvincular o foco do nutriente para o alimento em si.

Quanto à formação profissional para educação alimentar e nutricional: (i) promover projetos político-pedagógicos que enfoquem a educação alimentar e nutricional na formação de pedagogos e nutricionistas; (ii) comparar a educação alimentar e nutricional de hoje com a necessidade de repensar os preceitos filosóficos do curso de Nutrição, em especial a disciplina de Educação alimentar e nutricional; (iii) envolver a temática da educação alimentar e nutricional nas diversas disciplinas do curso de nutrição, tendo em vista que atualmente a mesma 
se restringe a disciplina específica; (iv) ter por foco da formação profissional do nutricionista o Direito Humano a Alimentação Adequada e a Segurança Alimentar e Nutricional.

Ao CONSEA: (i) ser o desencadeador e promotor de discussão sobre a educação alimentar e nutricional; (ii) pautar a educação alimentar e nutricional como tema central na próxima Conferência Nacional de Segurança Alimentar e Nutricional; (iii) fortalecer os CONSEAS nos estados e municípios como espaços de referência para segurança alimentar e nutricional.

Ao Estado: (i) regulamentar a publicidade de produtos alimentícios voltados para o público infantil e propor plano de comunicação em educação alimentar e nutricional; (ii) fomentar pesquisas sobre alimentação e nutrição nos diversos campos de conhecimento: antropologia, história, meio ambiente, psicologia etc.; (iii) priorizar a educação alimentar e nutricional em políticas públicas novas e nas já existentes; (iv) criar um programa de Escola Saudável que seja intersetorial; (v) criar políticas públicas articuladas e entre diversas áreas de proteção à saúde da população; (vi) criar política de abastecimento popular em que a educação alimentar e nutricional foque a produção de alimentos ecologicamente corretos; (vii) propor acordo social com as indústrias na elaboração de alimentos mais saudáveis e adequados; (viii) ampliar linhas de fomento em educação alimentar e nutricional para governo e sociedade civil.

Quanto aos espaços promotores de educação alimentar e nutricional: (i) preocupar-se com outros segmentos como os trabalhadores, comercial, indígenas e quilombolas; (ii) criar fóruns de discussão nos estados para discutir o formato da educação alimentar e nutricional e propor mecanismos de implantação e de troca de experiências; (iii) criar observatório de experiências em educação alimentar e nutricional na sociedade civil; (iv) pautar a educação alimentar e nutricional, no SUAS, nos Centros de Recuperação de Assistência Social (CRAS) e em conselhos que possuam relação com a temática.

Outras recomendações: (i) proteger o patrimônio genético alimentar das comunidades tradicionais; (ii) exemplificar estratégias para difundi-las (em âmbito ministerial) e dar subsídios para o município desenvolver suas ações; (iii) treinar multiplicadores de conhecimentos sobre alimentação e nutrição: política de educação permanente; (iv) mapear experiências no governo e na sociedade civil.

\section{Conclusão}

Diante dessa construção coletiva e debates promovidos, a Coordenação de Educação Alimentar e Nutricional encontra-se diante de novas perspectivas de atuação. Destaca-se a consolidação da educação alimentar e nutricional como estratégia para garantia e promoção da seguran- 
ça alimentar e nutricional, a atuação conjunta com os Programas Sociais do MDS, o apoio a projetos locais de educação alimentar e nutricional e o diagnóstico das ações de educação alimentar e nutricional desenvolvida no país.

\section{New Perspectives on Food and Nutrition Education}

Abstract: Considering the growing emphasis on food and nutrition education, as a result of the Zero Hunger strategy and the National Policy of Food and Nutrition, as well as the changes in the morbimortality of the population, it became fundamental to make reflections concerning food and nutrition education as a governmental action. In the Zero Hunger strategy, food and nutrition education is considered an structuring action, and consequently became, institutionally, part of the Ministry of Social Development and Fight Against Hunger (MDS), acting as an instrument to promote food and nutritional security. The Coordination responsible for developing educative actions in this Ministry has promoted two opportunities of reflection: and internal workshop, aiming to establish the institutional mark of food and nutrition education in MDS, and another workshop in the National Council of Food and Nutritional Security (CONSEA), aiming to subsidize a working group about healthy and adequate eating. As a result of these discussions emerged various recommendations and strategies as a base to educative actions in food and nutrition to be developed in the future, both by the government and the civil society. Among these recommendations we can highlight the importance of educative actions that promote autonomy for food choices, the interdisciplinary and transdisciplinary perspective for actions, respecting the different cultures and valuing the food history and regional diversity, recognizing the popular knowledge and respecting the local biodiversity.

Keywords: Public policies. Nutrition. Food and nutritional education.

\section{Nouvelles perspectives em Éducation Alimentaire et Nutricionnelle}

Resume: L'emphase croissante sur lê thème de l'education alimentaire et nutritionnelle, qui résulte de la stratégie Fome Zero et de la Politique Nationale d'Alimentation et de Nutrition, et aussi du changement de profil de morbimortalité de la population, a rendu fondamentale la réflexion sur sa configuration en tant qu'action governementale. Dans la stratégie Fome Zero, l'education alimentaire et nutritionnelle est prévue en tant qu'action structurante, de sorte qu'elle s'est configurée institutionnellement au Ministère du Développement Social et du Combat contre la Faim (MDS), et a comme perspective la promotion de la Sécurité Alimentaire 
et Nutritionnelle. Ainsi, la Coordination responsible par le développement d'actions éducatives dans ce Ministère a promudeux occasions de réflexion:I'une interne, qui visait à établir un nouveau repère institutionnel de l'éducation alimentaire et nutritionnelle dans le MDS et l'autre, dont l'objectif était d'appuyer le groupe de travail sur l'alimentation adéquate et saine du Conseil National de Sécurité Alimentaire. De ces discussions out émergé dímporantes recommandations et stratégies servant de base au développement d'actions éducatives en alimentation et nutrition, pour le govvernement et pour la société civile. Parmi ces recommandations, nous soulignos l'importance de développer des actions éducatives dans le sans de promouvioir láautonomie des individus, l'interdisciplnarité et la transdisciplinarité, tout en respectant les culture alimentaire, en valorisan l'hisoire alimentaire et la diversité régionale, qui recinnaissent en même temps les savoirs populaires et qui fomentet la biodiversité locale.

Mots-clés: Politiques publiques. Éducation alimentaire. Éducation alimentaire et nutritionnelle.

\section{Nuevas Perspectivas en Educación Alimentaria y Nutricional}

Resumen: El creciente énfasis en la temática de educación alimentaria y nutricional, resultado de la estrategia Hambre Cero (Fome Zero) y de la Política Nacional de Alimentación y Nutrición y el cambio en el perfil de morbimortalidad de la población, hizo necesario una reflexión sobre su configuración en lo referente a la acción gubernamental. En la estrategia Hambre Cero, la educación alimentaria y nutricional está prevista como una acción estructurante, de manera que la misma se configura institucionalmente en el Ministerio de Desarrollo Social y Combate al Hambre (MDS), teniendo como perspectiva la promoción alimentaria y nutricional. De este modo la Coordinación responsable por el desarrollo de acciones educativas en este Ministerio promovió dos oportunidades de reflexión: una interna que tenía como finalidad establecer el marco institucional de la educación alimentaria y nutricional en el MDS y la otra en el Consejo Nacional de Seguridad Alimentaria con el objetivo de ayudar al grupo de trabajo de alimentación adecuada y saludable. De estas discusiones surgieron importantes recomendaciones y estrategias como base para el desarrollo de acciones educativas en alimentación y nutrición tanto para el gobierno como para la sociedad civil. Entre esas recomendaciones, destacamos la importancia de que las acciones educativas sean desarrolladas en el sentido de promover la autonomía de los individuos, la interdisciplinaridad y la transdisciplinaridad, respetando las culturas alimentarias y valorando la historia alimentaria y la diversidad regional al mismo tiempo que se reconozcan los conocimientos populares y se fomente la biodiversidad local.

Palabras-clave: Políticas públicas. Educación alimentaria y nutricional. Nutrición. 


\section{Referências}

Batista Filho, M., Rissin, A. (2003). A transição nutricional no Brasil: tendências regionais e temporais. Cadernos de Saúde Pública, 19(Sup. 1), 181S-191S.

Brasil. Conselho Nacional de Segurança Alimentar e Nutricional. (2004). Relatório Final da II Conferência nacional de segurança alimentar e nutricional (CONSEA) (Versão 2). Brasília, DF: Autor.

Brasil. Ministério da Saúde. Instituto Nacional de Alimentação e Nutrição. (1990). Pesquisa nacional sobre saúde e nutrição: perfil de crescimento da população brasileira de 0 a 25 anos. Brasília, DF: Autor.

Brasil. Ministério do Desenvolvimento Social e Combate à Fome. Secretaria de Avaliação e Gestão da Informação. (2006). Chamada nutricional: um estudo sobre a situação das crianças do semi-árido brasileiro. Cadernos de Estudos: Desenvolvimento Social em Debate, (4), 1-105.

Demo, P. (2006). Formação. Recuperado em 12 de junho de 2006, de http://aprender.unb.br/mod/resource/view.php?id=21503

Instituto Brasileiro de Geografia e Estatística.(2006).Antropometria e análise do estado nutricional de crianças e adolescentes no Brasil: pesquisa de orçamentos familiares. Rio de Janeiro: Autor.

Wang, Y., Monteiro, C., Popkin, B. M. (2002). Trends of obesity and underweight in older children and adolescents in the United States, Brazil, China, and Russia. American Journal of Clinical Nutrition, 75, 971977.

Sabrina Ionata de Oliveira, Coordenadora Geral de Educação Alimentar e Nutricional, Ministério do Desenvolvimento Social e Combate a Fome - MDS, Quadra 02 Conjunto A-1 casa 35 - Sobradinho,Brasília - DF. CEP 73015-101. Endereço eletrônico: sabrina.ionata@gmail.com

Kathleen Sousa Oliveira, Coordenadora Técnica de Educação Alimentar e Nutricional Ministério do Desenvolvimento Social e Combate a Fome - MDS, QRSW 08 bloco B-3 apt. 305. Sudoeste.Brasília-DF. CEP: 70675-823. Endereço eletrônico: kathleen.ntr@terra.com.br

Recebido em: 30/04/2008

Aceito em: $27 / 11 / 2008$ 